\title{
Seasonal variation in drinking water concentrations of disinfection by-products in IZMIR and associated human health risks
}

\author{
Derya Baytak ${ }^{a, 1}$, Aysun Sofuoglu ${ }^{b}$, Fikret Inal ${ }^{c}$, Sait C. Sofuoglu ${ }^{b, *}$ \\ ${ }^{a} E$ Environmental Engineering Graduate Program, İzmir Institute of Technology, Gülbahçe, Urla 35430 İzmir, Turkey \\ bepartment of Chemical Engineering and Environmental Research Center, İzmir Institute of Technology, Gülbahçe, Urla 35430 İzmir, Turkey \\ cDepartment of Chemical Engineering, İzmir Institute of Technology, Gülbahçe, Urla 35430 İzmir, Turkey
}

\section{A R T I C L E D A T A}

\section{Article history:}

Received 26 May 2008

Received in revised form 30 July 2008

Accepted 11 August 2008

Available online 20 September 2008

Keywords:

Trihalomethanes

Haloacetonitriles

Carcinogenic risk

Noncarcinogenic risk

Tap water

\begin{abstract}
A B S T R A C T
Seasonal variation in concentrations of two different disinfection by-product groups, trihalomethanes (THMs) and haloacetonitriles (HANs), was investigated in tap water samples collected from five sampling points (one groundwater and four surface water sources) in İzmir, Turkey. Estimates of previously published carcinogenic and noncarcinogenic risks through oral exposure to THMs were re-evaluated using a probabilistic approach that took the seasonal concentration variation into account. Chloroform, bromoform, dibromochloromethane and dichloroacetonitrile were the most frequently detected compounds. Among these, chloroform was detected with the highest concentrations ranging from 0.03 to $98.4 \mu \mathrm{g} / \mathrm{L}$. In tap water, at the groundwater supplied sampling point, brominated species, bromoform and dibromoacetonitrile, were detected at the highest levels most probably due to bromide ion intrusion from seawater. The highest total THM and total HAN concentrations were detected in spring while the lowest in summer and fall. The annual average total THM concentration measured at one of the surface water supplied sampling points exceeded the USEPA's limit of $80 \mu \mathrm{g} / \mathrm{L}$. While all non-carcinogenic risks due to exposure to THMs in İzmir drinking water were negligible, carcinogenic risk levels associated with bromodichloromethane and dibromochloromethane were higher than one in million.
\end{abstract}

(c) 2008 Elsevier B.V. All rights reserved.

\section{Introduction}

Chlorination of drinking water leads to formation of disinfection by-products (DBPs) which may create adverse health effects on human beings. The DBPs are formed as a result of reactions between the precursor materials (natural organic matter and bromide ion) and aqueous forms of the disinfectants. The major groups of DBPs are trihalomethanes (THMs), haloacetic acids (HAAs), haloacetonitriles (HANs), and halogenated ketones (HKs). THMs include four species; chloroform (CF), bromodichloromethane (BDCM), dibromochloromethane (DBCM) and bromoform (BF). Epidemiological studies have suggested that exposure to DBPs increases the risk of bladder, colon-rectum, leukemia, stomach and rectal cancers as well as abortion, low birth weight, and birth defects (IARC, 1991; Calderon, 2000; Villanueva et al., 2004).

The formation of DBPs is affected by several factors including water temperature and $\mathrm{pH}$, nature and concentration of the

\footnotetext{
* Corresponding author. Tel.: +90 232750 6648; fax: +90 2327506645. E-mail addresses: cemilsofuoglu@iyte.edu.tr, saitcemil@iit.edu (S.C. Sofuoglu).

${ }^{1}$ Current address: Department of Chemical Engineering, İzmir Institute of Technology, Gülbahçe, Urla 35430 İzmir, Turkey.
} 
natural organic matter (NOM), concentration of bromide ion, disinfectant type and dose, and residence time of water in the distribution system. The concentration of NOM is the most significant parameter affecting DBP formation (Liang and Singer, 2003; Ates et al., 2007). The temperature and pH also affect the reaction rates of chlorine depletion in water, and aqueous stability of DBPs (Villanova et al., 1997; Glezer et al., 1998; Kim et al., 2002). In addition, increase in the bromide ion concentration shifts the type of DBPs from chlorinated compounds to brominated ones (Heller and Grossman, 1999; Kampioti and Stephanou, 2002). The levels of the aforementioned factors vary seasonally. Therefore, consumers receive drinking water with a variable quality throughout a year in terms of DBP concentrations.

Among the THMs, chloroform is the most frequently detected compound with a concentration range of 2-228 $\mu \mathrm{g} / \mathrm{L}$ (Rodriguez et al., 2003). However, depending on the bromide content of raw water, the concentration of bromoform may be greater than chloroform (Westerhoff et al., 2004). The other groups of DBPs are generally detected at lower concentrations than THMs. Many studies on seasonal variation of THMs suggested that these compounds tend to be higher in summer since organic matter content of water source increases (Williams et al., 1998; Rodriguez et al., 2004). A recent study reported that (Ates et al., 2007) 16 of the 29 surface source waters of Turkey had the highest THM formation potential during winter, whereas six source waters had it in spring and autumn. There was no general trend for seasonal variation in formation potentials because there may be temporal and spatial variation in factors such as structure of NOM and reactivity of chlorine. Because the reaction between free residual chlorine and natural organic matter continue throughout the distribution system, and chlorine is dosed at certain intervals as a protection against waterborne diseases, the highest THM concentrations were also detected in water samples taken near the end of the drinking water distribution systems (Lebel et al., 1997; Rodriguez and Serodes, 2001).

In Turkey, a couple of studies were conducted to investigate the seasonal and spatial variation in THM concentrations in drinking water (Tokmak et al., 2004; Toroz and Uyak, 2005). The levels of THMs in tap water samples from Ankara and Istanbul were found to be the highest in summer, although THM formation potential of the source waters was found to be highest in winter and autumn for Ankara and Istanbul, respectively, in the laboratory at constant temperature (Ates et al., 2007). This discrepancy may have risen due to the fact that all were conducted in different years. In addition, THM concentrations tend to increase with the residence time of water in the distribution system. However, there is no study that reports HAN levels in Turkey, which are found to be more toxic than regulated carbon based DBPs such as the HAAs (Muellner et al., 2007). In our recent study, which was conducted to investigate volatile organic compound levels in drinking water of Izmir, carcinogenic risk levels were found to be greater than the acceptable level for brominated THMs even at concentrations that were in attainment of drinking water standards (Kavcar et al., 2006).

A maximum contaminant level (MCL) of $150 \mu \mathrm{g} / \mathrm{L}$ is set for total trihalomethanes (TTHM) in Turkey and this level will be lowered to $100 \mu \mathrm{g} / \mathrm{L}$ by the year 2012 (Turkish Ministry of Health, 2005). The US Environmental Protection Agency (USEPA) proposed an MCL of $80 \mu \mathrm{g} / \mathrm{L}$ for TTHM under Stage 1 of the Disinfectant/Disinfection By-Product (D/DBP) Rule, based on annual average of four samples collected quarterly (USEPA, 1998). According to Stage 2 D/DBP rule, at each sampling location the average concentration of TTHM must meet the MCL.

There are several studies that estimated carcinogenic and non-carcinogenic risk levels for THMs (Hsu et al., 2001; Sofuoglu et al., 2003; Lee et al., 2004; Wang et al., 2007) other than our previous study (Kavcar et al., 2006). In these studies, noncarcinogenic risks were found to be lower than the demarcation value. The highest carcinogenic risks were calculated for BDCM as $1.25 \times 10^{-5}$ and $6.82 \times 10^{-5}$ in Canada (Wang et al., 2007) and Hong Kong (Lee et al., 2004), respectively. In Taiwan (Hsu et al., 2001) and Arizona, USA (Sofuoglu et al., 2003), the highest risks were estimated for chloroform and the risk levels were $1.8 \times 10^{-4}$ and $2.1 \times 10^{-7}$, respectively, however, oral ingestion cancer risk factor for chloroform has been withdrawn (IRIS, 2007).

Although our previous study gathered information about THM concentrations in drinking water samples across the metropolitan area of İzmir, seasonal variation was not investigated. A study on the seasonal variation of DBP levels was needed to enhance the estimated risk levels. This study aimed to investigate seasonal and spatial variations in concentrations of THMs, HANs and HKs in drinking water of İzmir. The population carcinogenic and non-carcinogenic risk distributions were reconstructed with the enhanced data set.

\section{Materials and methods}

\subsection{Sampling procedure}

Drinking water samples for DBP analysis were collected at Konak (Hatay), Balçova, Narlıdere, Güzelbahçe and Urla districts (Fig. 1). Samples were collected between July 2006 and April 2007 from tap water at one sampling point in each district every six days. Among sampling locations, Urla is $30 \mathrm{~km}$ away from the metropolitan İzmir and represented a non-urban area whereas Konak, Balçova, Narlıdere are metropolitan districts, and Güzelbahçe is a suburban area. Sampling points in the urban and suburban area were served from two different drinking water sources. Konak district supplies drinking water from Tahtalı Reservoir, while Balçova, Narlıdere, and Güzelbahçe districts from Balçova Reservoir.

Water supplied from Tahtalı Reservoir is treated in Tahtalı Drinking Water Treatment Plant (TDWTP) and distributed to Hatay, Buca, Karabağlar, and Yeşilyurt regions. TDWTP has a capacity of $520,000 \mathrm{~m}^{3} /$ day, and consists of aeration, coagulation, flocculation-sedimentation, filtration, chlorination, and filter press units. Pre-chlorination is applied to aerated water, and final chlorination is applied to water before leaving the plant. The drinking water of Balçova Dam is treated in Balçova Drinking Water Treatment Plant (BDWTP), which has a capacity of $70,000 \mathrm{~m}^{3} /$ day. The plant has aeration, prechlorination, rapid-sand filters, and final chlorination units. Balçova, Narlıdere and Güzelbahçe sampling points are on the same main line of the BDWTP distribution system. The drinking water source of the Urla district is groundwater.

A total of 44 samples were collected at each sampling point. These samples were analyzed for THMs which include CF, 


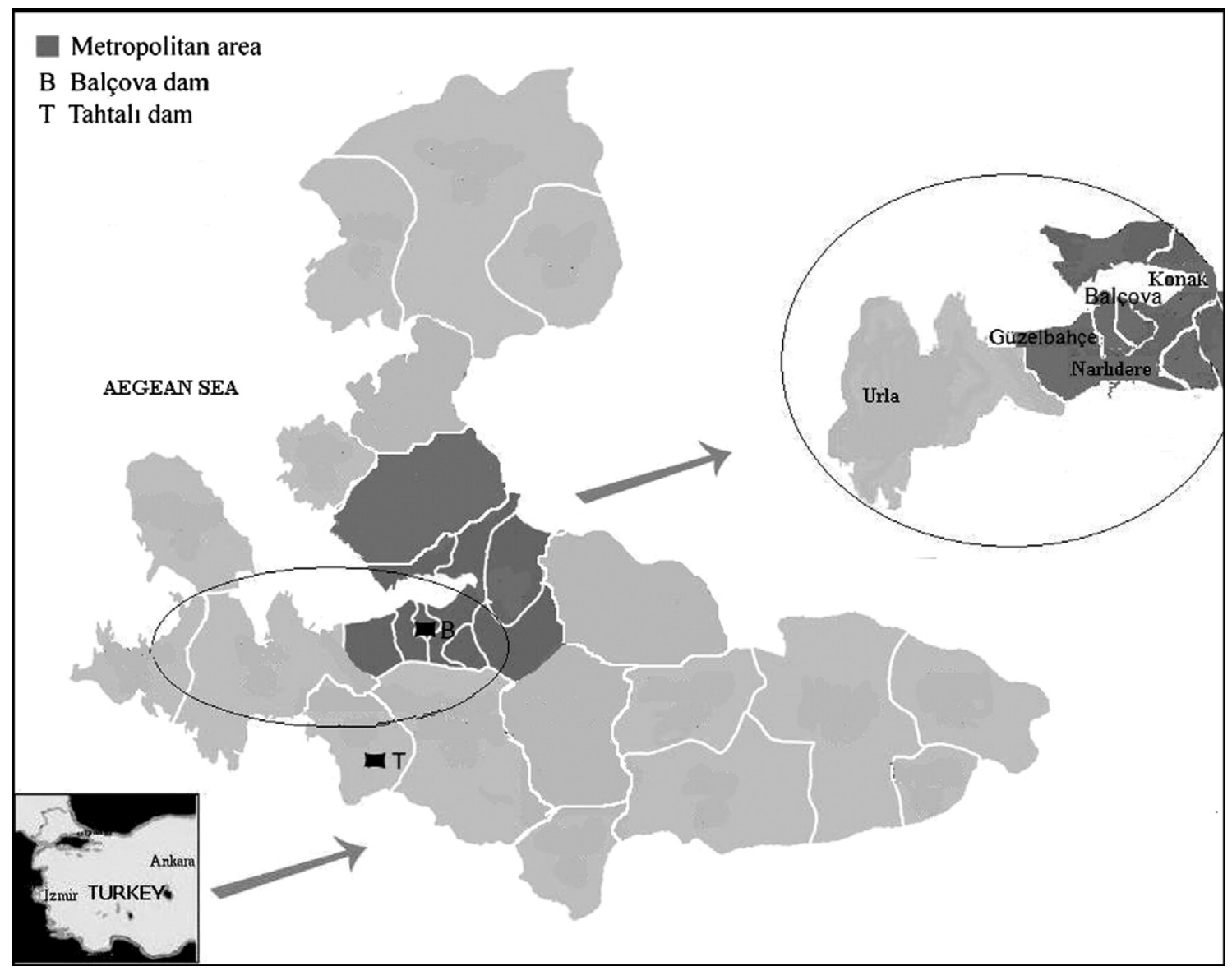

Fig. 1-Map of the sampling locations.

DBCM, BDCM and BF, HANs which include dichloroacetonitrile (DCAN), bromochloroacetonitrile (BCAN), dibromoacetonitrile (DBAN) and trichloroacetonitrile (TCAN), and HKs which include 1,2-dichloropropane (1,2-DCP) and trichloropropane (1,1,1-TCP). In addition, samples collected at Urla sampling point and Tahtalı Reservoir (raw) water were analyzed for bromide ion. Samples were also collected from inlet and outlet of the Tahtalı Drinking Water Treatment Plant (TDWTP), and analyzed for Non-Purgeable Organic Carbon (NPOC). Sampling at the TDWTP was carried out by İzmir Water and Sewerage Authority (IZSU), the samples were then sent to our laboratory for NPOC and bromide ion analyses. All BDWTP operational parameter data were obtained from IZSU.

Sampling was performed in public buildings using a faucet of the washroom nearest to the street. Before collecting the samples, the system was flushed for about three minutes. Samples were collected in 40-mL pre-cleaned screw cap amber glass vials with polypropylene cap and silicone septa (Supelco) for DBP analysis. Vials were washed with detergent (Alconox), rinsed first with tap water then three times with ultra pure chemical free MilliQ (Millipore Elix-5/Milli-Q) water. Finally, they were rinsed with GC grade acetone (Merck) and placed in an oven at $105{ }^{\circ} \mathrm{C}$ for an hour. Before sampling, $0.8 \mathrm{~g}$ of the mixture of $1 \%$ sodium phosphate monobasic $\left(\mathrm{NA}_{2} \mathrm{HPO}_{4}\right)$ and $99 \%$ potassium phosphate monobasic $\left(\mathrm{KH}_{2} \mathrm{PO}_{4}\right)$ by weight was added to vials to lower the sample $\mathrm{pH}$ to between 4.8 and 5.5 in order to inhibit base catalyzed degradation of the HANs, and to standardize the $\mathrm{pH}$ of the samples. Then, $0.004 \mathrm{~g} \mathrm{NH}_{4} \mathrm{Cl}$ (Merck) was added to provide $100 \mathrm{mg} / \mathrm{L}$ in each vial to eliminate any remaining residual chlorine to stop further DBP formation. The above reagents were heated at $105^{\circ} \mathrm{C}$ for one hour before use. Once collected, samples were stored in the dark at $4^{\circ} \mathrm{C}$, and transported to the laboratory for analytical procedures. At each time, free residual chlorine was measured in the field by using a DPD (N, N-Diethyl-P-Phenylenediamine) test kit (Lovibond, PC Checkit 60684). For NPOC analysis, samples were also collected in 40-mL amber glass vials with screw cap, and acidified with $2 \mathrm{~mL} \mathrm{98 \%} \mathrm{H}_{2} \mathrm{SO}_{4}$. Samples for bromide analysis were collected in 60-mL HDPE bottles (Nalgene). The water quality and operational parameters such as $\mathrm{pH}$, temperature and chlorine dose were obtained from the routine measurements of the treatment plants. The data from BDWTP were available for only a part of the sampling campaign (July to August and December to February) because the plant was not in operation throughout the sampling campaign.

\subsection{DBP analysis}

In this study, the USEPA method 551.1 was followed for the analysis of THMs, HANs, HKs, and chloropicrin (CP) (USEPA, 1995). After the liquid-liquid extraction, analyses of DBPs were performed by using a gas chromatograph (GC) equipped with an electron capture detector (Agilent 6890N) and auto sampler (Agilent 7893). 
THM calibration mix (Supelco) was purchased as $2000 \mu \mathrm{g} / \mathrm{L}$ in acetone whereas halogenated volatiles mix (HANs, HKs and CP) (Supelco) was $2000 \mu \mathrm{g} / \mathrm{L}$ in methanol. These standards were prepared in acetone, in 2-mL crimp capped vials to achieve minimum headspace, and stored in the dark in a freezer at $-27{ }^{\circ} \mathrm{C}$. Procedural calibration standards were prepared, and extracted in exactly the same manner as a sample to compensate for any inefficiency in the procedure. The calibration standards were $2,10,20,50$, and $100 \mu \mathrm{g} / \mathrm{L}$ for THMs; $0.25,1,5,10$, and $25 \mu \mathrm{g} / \mathrm{L}$ for HANs, HKs and CP. The $R^{2}$ values for the linearized calibration curves were between 0.979 and 0.999 for all DBPs.

\subsection{Non-purgeable organic carbon analysis}

NPOC measurements were carried out by a Shimadzu TOC$\mathrm{V}_{\mathrm{CPH}}$ analyzer with OCT-1 Shimadzu sampler. In order to obtain the calibration curves, the stock standard solution for total carbon was prepared by dissolving $2.125 \mathrm{~g}$ potassium hydrogen phthalate $\left(\mathrm{C}_{8} \mathrm{H}_{5} \mathrm{KO}_{4}\right)$ in $1000 \mathrm{~mL}$ ultra pure water. Then, the stock solution was diluted in appropriate amounts with ultra pure water with concentrations of $1,5,10,20$, and $50 \mathrm{mg} / \mathrm{L}$.

\subsection{Bromide ion analysis}

The concentration of bromide ion was measured according to the USEPA method 300 employing an ion chromatography system (Dionex) which included an electrochemical detector (ED 50), a pump (GP 50 gradient), an analytical column (AS9$\mathrm{HC}$, Ionpac). Eluent composition was $10 \mathrm{mM} \mathrm{Na}_{2} \mathrm{CO}_{3}$ with a flow rate of $1 \mathrm{~mL} / \mathrm{min}$. External bromide standards with concentrations $0.5,2.5,5,10$, and $20 \mathrm{mg} / \mathrm{L}$ were prepared from a standard that included $100 \mathrm{mg} / \mathrm{L}$ bromide ion (Dionex). The detection limit of the method was $0.15 \mu \mathrm{g} / \mathrm{L}$.

\subsection{Quality control/quality assurance procedure (QA/QC)}

Laboratory requirements included, initial demonstration of laboratory capability, determination of method detection limit, analysis of laboratory reagent blanks, field reagent blank, field duplicates and calibration check standards (USEPA, 1995).

For the initial demonstration of the laboratory capability, the observed chromatographic peaks, obtained by running a standard solution of method analytes were identified by comparing the retention times with those given in the EPA method 551. Then, oven temperature program was modified according to retention time of the last peak of the method analyte. To obtain a smooth baseline, a non-polar organic solvent (hexane) was run before analysis of the each batch of samples. Before each run, the GC syringe was rinsed three times with ultra pure water and acetone, respectively.

The field reagent blanks were collected to determine if any interference was present in the field environment. Laboratory reagent blanks were analyzed to determine if method analytes or other interferences were present in the laboratory environment, the reagents, or the apparatus. On the other hand, the precision of the measurements was estimated using field duplicates (FD). The relative percent difference (RPD) between two parallel samples was calculated according to Eq. (1). The average RPD for TTHM, total haloacetonitriles (THAN), HKs, and CP were calculated as $14 \%, 28 \%, 25 \%$, and $26 \%$, respectively. Even though the RPD for THANs, HKs, and CP were higher than the literature values, these compounds were detected at very low concentrations (i.e., detection limit of instrument).

$\mathrm{RPD}(\%)=\frac{(\mathrm{FD} 1-\mathrm{FD} 2)}{1 / 2(\mathrm{FD} 1+\mathrm{FD} 2)} \times 100$

The method detection limits were calculated for each compound according to Eq. (2) by analyzing seven replicates of standard solution at a concentration of $0.05 \mu \mathrm{g} / \mathrm{L}$ for HANs, HKs and CP, and $0.25 \mu \mathrm{g} / \mathrm{L}$ for THMs.

$\mathrm{MDL}=\mathrm{SD} \times \mathrm{t}_{\alpha / 2, n-1}$

where SD is the standard deviation of the mean for seven replicate samples and t-value is the Student's t-value for $99 \%$ confidence level and $n-1$ degrees of freedom. The method detection limits and the recoveries for the method analytes are shown in Table 1.

Continuing calibration checks were performed every 20 samples. If the relative percent difference between response of the initial calibration and the calibration check standard was $>20 \%$, the instrument was considered as out of calibration, and recalibrated.

\subsection{Statistical methods}

Normality of the data was checked with Kolmogorov-Smirnov test. Non-normality was the case for each of the concentration sets. Non-parametric statistical tests such as, Mann-Whitney and Kruskal-Wallis, were used to investigate whether the concentrations of DBPs differed depending upon water source and seasons. Mann-Whitney test was used to test whether two independent samples are from the same population. In order to compare more than two independent samples Kruskal-Wallis test was used.

The $p$-values, the probability of error in accepting the observed result as valid, obtained by non-parametric tests, are compared with the chosen significance level $(\alpha=0.05) . p<0.05$, indicating the medians are different with a probability of $95 \%$, deemed a statistically significant difference between the compared samples.

Table 1 - Method detection limits and recoveries for DBPs

\begin{tabular}{lcc} 
Compound & MDL $(\mu \mathrm{g} / \mathrm{L})$ & Recovery $(\%)$ \\
\hline CF & 0.030 & 99 \\
TCAN & 0.009 & 50 \\
DCAN & 0.002 & 108 \\
BDCM & 0.057 & 87 \\
DCP & 0.026 & 135 \\
CP & 0.015 & 74 \\
DBCM & 0.014 & 103 \\
BCAN & 0.018 & 76 \\
TCP & 0.009 & 109 \\
BF & 0.044 & 139 \\
DBAN & 0.073 & 62 \\
\end{tabular}




\subsection{Exposure and risk assessment}

Based on the THM data obtained in this study along with those measured in our previous study (Kavcar et al., 2006), a human health risk assessment was conducted to determine carcinogenic and non-carcinogenic risks due to exposure to THMs via ingestion pathway.

For calculation of lifetime daily exposure for ingestion pathway the following equation, which is modified from the USEPA (1992), was used:

$\mathrm{CDI}=\frac{\mathrm{C} \times \mathrm{DI}}{\mathrm{BW}}$

where $C$ is the drinking water contaminant concentration $(\mathrm{mg} / \mathrm{L})$, DI is the average daily intake rate of drinking water $(\mathrm{L} / \mathrm{d})$, BW is the body weight in $\mathrm{kg}$, and, CDI is the chronic daily intake $(\mathrm{mg} / \mathrm{kg} / \mathrm{d})$. DI and BW data for İzmir population were obtained from the questionnaires administered in our previous study (Kavcar et al., 2006). Lifetime cancer risk associated with ingestion exposure was calculated using the following equation (USEPA, 2005).

$\mathrm{R}=\mathrm{CDI} \times \mathrm{SF}$

where $R$ is the probability of excess lifetime cancer risk, CDI is the chronic DI $(\mathrm{mg} / \mathrm{kg} / \mathrm{d})$, and SF is the slope factor of the chemical $(\mathrm{mg} / \mathrm{kg} / \mathrm{d})^{-1}$.

The non-cancer risk levels, the hazard quotient (HQ) levels, were calculated using the following equation (USEPA, 1999):

$\mathrm{HQ}=\frac{\mathrm{CDI}}{\mathrm{RfD}}$

where RfD is the reference dose $(\mathrm{mg} / \mathrm{kg} / \mathrm{d})$. SF and RfD values for THMs were obtained from the USEPA (IRIS, 2007). The SF values were $6.2 \times 10^{-6}, 8.4 \times 10^{-2}$, and $7.9 \times 10^{-2}$ for BDCM, DBCM and $\mathrm{BF}$, respectively. The RfD values were $1 \times 10^{-2}$ for $\mathrm{CF}$ and $2 \times 10^{-2}$ for BDCM, DBCM and BF.

All risk levels were estimated probabilistically by Monte Carlo simulation method using Crystal Ball software (v 4.0e). Monte Carlo simulation is a computer-based method of analysis that uses statistical sampling techniques in obtaining a probabilistic approximation to the solution of a mathematical equation or model (USEPA, 1997). Best fitting probability distributions are determined for each variable in Eq. (3) using the simulation software. These probability distributions were used as the input distributions for the prediction of the exposure and risk levels. The risk estimates were expressed as probability distribution of values. During a single trial, values were selected randomly from the defined possibilities for each uncertain variable, and then the output of the model was calculated. To obtain a representative risk distribution, the simulation was run for 10,000 trials, and 10,000 forecasts (or possible outcomes) were obtained.

\section{Results and discussion}

\subsection{Water quality and operational parameters}

Chlorine doses and drinking water quality parameters including NPOC concentration, $\mathrm{pH}$, and temperature were measured in samples from both TDWTP and BDWTP. NPOC concentrations in the raw water of both of the plants were below $5 \mathrm{mg} / \mathrm{L}$ which is typical for unpolluted fresh-surface waters (Table 2). In support, the annual average dissolved organic carbon concentrations of the raw water of TDWTP and BDWTP were reported as 3.06 and $1.80 \mathrm{mg} / \mathrm{L}$, respectively, in 2004 (Ates et al., 2007).

The number of samples from BDWTP, in this study, was less than TDWTP because the plant supplied water to the system for only five months of the sampling period. It should also be noted that, the mean NPOC concentration for BDWTP raw water represented only three months (December, January and February) since the NPOC were not measured for the other months. Comparing the median NPOC concentrations of the two plants measured in the three month period in which both plants were in operation, showed that the differences in the concentrations were not statistically significant $(p=0.35)$. Moreover, the mean concentrations of pre- and final chlorine doses applied at TDWTP were about three times higher than that of BDWTP overall and in the five-month period in which both plants were in operation. Bromide ion concentrations were measured in the raw water of TDWTP and tap water at Urla sampling site. Annual average raw water bromide ion concentration was found to be $0.24 \mathrm{mg} / \mathrm{L}$ for TDWTP, while it was $1.9 \mathrm{mg} / \mathrm{L}$ in Urla. The high occurrence of bromide ion in Urla may be related to intrusion of seawater to the groundwater since this district is located on the coast. Although BDWTP raw water bromide ion concentrations were not measured, it has previously been reported to be lower than $0.02 \mathrm{mg} / \mathrm{L}$ (Ates et al., 2007).

Seasonal variation in TDWTP raw water NPOC concentrations is shown in Fig. 2. Although the mean concentration of

Table 2 - Descriptive statistics for operational and drinking water quality parameters

Parameter

\begin{tabular}{lcccccc}
\cline { 2 - 6 } & $\mathrm{N}$ & Mean & Med. ${ }^{\ddagger}$ & SD & Min & Max \\
\hline NPOC $^{*}$ & 43 & 3.76 & 3.76 & 0.46 & 3.05 & 5.06 \\
NPOC $^{* *}$ & 42 & 3.20 & 3.18 & 0.46 & 2.45 & 4.59 \\
Temperature $^{*}$ & 43 & 16.9 & 16.9 & 5.14 & 9.60 & 24.4 \\
pH $^{*}$ & 43 & 7.87 & 7.87 & 0.27 & 7.50 & 8.70 \\
PreCl $_{2}$ dose & 48 & 3.34 & 3.34 & 1.36 & 1.36 & 6.01 \\
FinalCl $_{2}$ dose & 48 & 1.02 & 1.02 & 0.16 & 0.83 & 1.74 \\
\hline
\end{tabular}

${ }^{*}$ Values are for raw water, ${ }^{* *}$ values are for treated water, ${ }^{*}$ median.

$\mathrm{N}$ : sample size, SD: standard deviation, NPOC concentrations and chlorine doses are in $\mathrm{mg} / \mathrm{L}$.
Balçova drinking water treatment plant

\begin{tabular}{rccccc}
\hline N & Mean & Med. ${ }^{\ddagger}$ & SD & Min & Max \\
9 & 3.55 & 3.56 & 0.48 & 2.85 & 4.31 \\
9 & 3.50 & 3.36 & 0.85 & 2.62 & 4.89 \\
16 & 10.9 & 10.3 & 1.97 & 7.70 & 14.2 \\
16 & 8.13 & 8.13 & 0.38 & 7.52 & 8.64 \\
23 & 0.75 & 0.75 & 1.11 & 0.36 & 5.50 \\
23 & 0.29 & 0.29 & 0.09 & 0.16 & 0.70
\end{tabular}




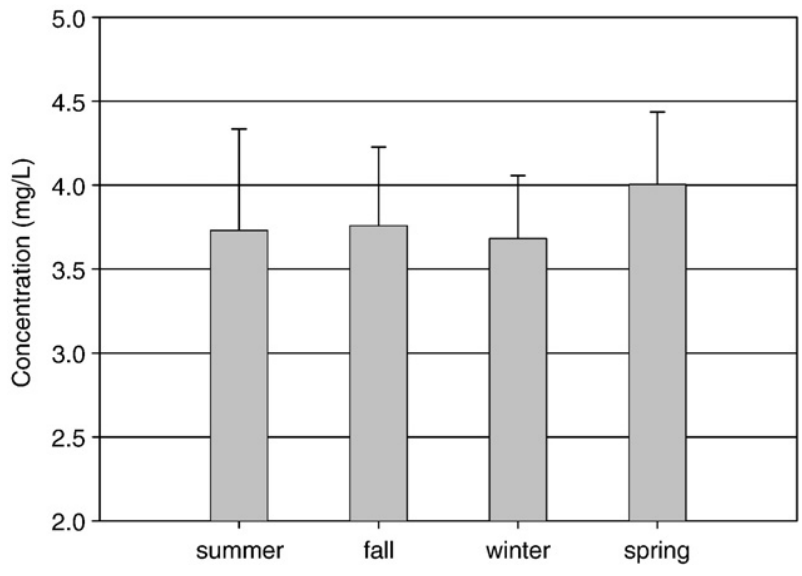

Fig. 2-Seasonal variation in the raw water NPOC concentrations at Tahtalı Drinking Water Treatment Plant (error bars show one standard deviation).

summer, fall, and winter were comparable, the maximum concentration levels were measured in summer $(5.1 \mathrm{mg} / \mathrm{L})$ and fall $(4.6 \mathrm{mg} / \mathrm{L})$. Differences in the mean NPOC concentrations in spring and the remaining seasons were not significant $(p>0.19)$. The reason for the stable NPOC level in the raw water was, possibly, climatic conditions during the study period, which resulted in unusually high temperatures in winter and low precipitation throughout Turkey.

\subsection{DBP concentrations}

Descriptive statistics of analyzed DBPs are presented in Table 3. Among all DBPs CF, BF, DBCM, and DCAN were detected at the highest frequencies ( $\geq 99 \%)$ while TCAN and CP were detected at the lowest frequencies ( $\leq 70 \%)$. The ranges for all compounds were very large because while very low concentrations depicted the non-urban site, very high concentrations were the case for one of the urban sites. CF was the most abundant DBP with a concentration range of $0.03-98 \mu \mathrm{g} / \mathrm{L}$. Concentrations of HANs were found to be much lower than THMs, which is in agreement with the literature (Simpson and Hayes, 1998; Kim et al., 2002).
The mean THAN concentration of $13 \mu \mathrm{g} / \mathrm{L}$ was higher than the concentrations measured in Melbourne, Australia $(2-7 \mu \mathrm{g} / \mathrm{L}$, Simpson and Hayes, 1998), and Hyogo Prefecture, Japan (1.6$3.2 \mu \mathrm{g} / \mathrm{L}$, Kawamoto and Makihata, 2004). The mean concentrations of 1,2-DCP and 1,1,1-TCP were found to be lower than the other DBPs except for CP, as suggested by Golfinopoulos and Nikolaou (2005). No drinking water concentration levels were reported for HANs and HKs for Turkey in the literature. The mean TTHM level $(48.5 \mu \mathrm{g} / \mathrm{L})$ falls in the range of TTHM levels measured in Australia (Simpson and Hayes, 1998) and Spain (Villanueva et al., 2003). The mean concentrations of THMs in summer and fall were lower while the mean concentration of THMs in spring was higher than the levels reported for Istanbul, Turkey (Toroz and Uyak, 2005).

The five selected sampling points in this study represented three different water sources. While Tahtalı and Balçova Reservoirs are surface waters, the drinking water source of the Urla district is groundwater. The highest concentrations of TTHM, THAN and HK were measured at Hatay, where drinking water is supplied from Tahtalı Reservoir (Table 4). Below, reservoir names are used for the sampling points that represent them. The lowest concentrations were measured in Urla. All DBP concentrations in Urla were below $20 \mu \mathrm{g} / \mathrm{L}$ since the source of the water is groundwater, which has much lower organic matter content compared to surface waters, being naturally protected from runoff waters. All comparisons made for the variation by source in this section are based on the five-month period in which both plants were in operation. The mean, median, minimum, and maximum DBP concentrations for Tahtalı were found to be higher than those for Balçova reservoir. The high occurrence of DBPs for Tahtall reservoir may be related to relatively higher NPOC concentrations in the raw water, as well as the high pre- and post-chlorine doses applied during drinking water treatment. These findings were also in agreement with the results of Ates et al. (2007) who reported $87 \mu \mathrm{g} / \mathrm{L}$ and $63 \mu \mathrm{g} / \mathrm{L}$ annual average concentrations of TTHM in water samples from Tahtalı and Balçova reservoirs, respectively, chlorinated at the laboratory.

CF was the most abundant THM compound followed by BDCM, DBCM and BF, for samples from Tahtalı and Balçova reservoirs (Fig. 3); a result in agreement with the literature (Ates et al., 2007). The composition was similar for the two

Table 3 - Descriptive statistics for DBP concentrations in Izmir drinking water

\begin{tabular}{|c|c|c|c|c|c|c|c|c|c|}
\hline DBPs & $N$ & $F$ & Median & Mean & $\mathrm{SD}$ & Min & $\operatorname{Max}$ & 90th percentile & 95th percentile \\
\hline $\mathrm{CF}$ & 221 & 99 & 22.0 & 22.0 & 18.4 & 0.03 & 98.4 & 47.7 & 55.2 \\
\hline BDCM & 177 & 83 & 13.0 & 10.3 & 9.02 & 0.01 & 43.8 & 22.3 & 28.9 \\
\hline DBCM & 221 & 100 & 8.39 & 14.7 & 12.7 & 0.19 & 65.9 & 31.9 & 38.9 \\
\hline $\mathrm{BF}$ & 221 & 99 & 2.77 & 4.45 & 4.33 & 0.04 & 19.1 & 12.2 & 14.2 \\
\hline TTHM & 221 & - & 46.3 & 48.5 & 35.8 & 2.86 & 183 & 99.5 & 124.8 \\
\hline DCAN & 221 & 99 & 3.97 & 3.59 & 4.12 & 0.00 & 20.8 & 8.77 & 13.3 \\
\hline BCAN & 221 & 95 & 2.26 & 3.2 & 2.74 & 0.01 & 11.9 & 7.42 & 8.24 \\
\hline DBAN & 217 & 95 & 2.77 & 4.23 & 3.62 & 0.00 & 16.4 & 9.72 & 11.4 \\
\hline TCAN & 178 & 67 & 0.05 & 2.49 & 6.35 & 0.00 & 54.6 & 7.69 & 14.6 \\
\hline THAN & 217 & - & 10.1 & 13.1 & 10.8 & 0.25 & 88.4 & 27.4 & 33.6 \\
\hline 1,2-DCP & 177 & 81 & 0.45 & 0.60 & 0.75 & 0.01 & 7.82 & 1.16 & 1.59 \\
\hline 1,1,1-TCP & 177 & 80 & 1.61 & 1.88 & 1.50 & 0.01 & 7.81 & 3.66 & 4.66 \\
\hline HKs & 177 & - & 2.07 & 2.48 & 2.25 & 0.02 & 15.6 & 4.82 & 6.25 \\
\hline $\mathrm{CP}$ & 177 & 70 & 0.17 & 2.48 & 1.91 & 0.08 & 9.33 & 4.95 & 6.00 \\
\hline
\end{tabular}

All values are in $\mu \mathrm{g} / \mathrm{L}, \mathrm{N}$ : sample size, F: detection frequency (\%), SD: standard deviation. 
Table 4 - Descriptive statistics for DBP concentrations across water sources

\begin{tabular}{|c|c|c|c|c|c|c|c|c|c|}
\hline DBPs & $S^{a}$ & $\mathrm{~N}$ & Mean & Median & SD & Min & $\operatorname{Max}$ & 90th percentile & 95th percentile \\
\hline \multirow[t]{3}{*}{ TTHM } & 1 & 21 & 89.0 & 84.2 & 20.3 & 40.0 & 124.9 & 115.3 & 124.0 \\
\hline & 2 & 21 & 45.8 & 44.4 & 16.8 & 19.9 & 87.4 & 68.6 & 85.6 \\
\hline & 3 & 18 & 12.4 & 12.7 & 3.56 & 4.93 & 18.4 & 16.8 & 18.1 \\
\hline \multirow[t]{3}{*}{ THAN } & 1 & 21 & 20.1 & 16.4 & 10.4 & 7.36 & 43.6 & 38.3 & 43.1 \\
\hline & 2 & 21 & 12.7 & 11.6 & 9.16 & 2.85 & 41.2 & 21.9 & 39.3 \\
\hline & 3 & 18 & 4.86 & 5.78 & 3.5 & 0.24 & 10.4 & 9.73 & 10.2 \\
\hline \multirow[t]{2}{*}{ HKs } & 1 & 21 & 4.10 & 4.32 & 2.43 & 0.56 & 9.06 & 7.40 & 8.90 \\
\hline & 2 & 21 & 2.73 & 2.96 & 1.59 & 0.33 & 5.46 & 4.91 & 3.86 \\
\hline
\end{tabular}

${ }^{a}$ Water source, N: sample size, SD: standard deviation.

All concentrations are in $\mu \mathrm{g} / \mathrm{L}$.

(1) Tahtalı Reservoir.

(2) Balçova Reservoir

(3) Ground Water.

reservoirs with approximately $51 \%, 25 \%, 20 \%$ and $4 \%$, for $\mathrm{CF}$, BDCM, DBCM and BF, respectively. However, CF was found to be the major THM specie in drinking water of Ankara, 90-95\% of the TTHM (Tokmak et al., 2004) probably due to low bromide ion levels in raw water. When the mean concentrations of four THM species were compared with those reported for İstanbul tap water (Toroz and Uyak, 2005), brominated THM species were found to be lower in İzmir, although the annual average concentration of bromide ion in İzmir $(0.26 \mathrm{mg} / \mathrm{L})$ was higher than that of İstanbul $(0.19 \mathrm{mg} / \mathrm{L})$.

In Urla, because of extremely high bromide ion levels (1.1$3.4 \mathrm{mg} / \mathrm{L}$ ), concentration of BF contributed about $95 \%$ to the TTHM. However, for Hatay samples, concentrations of BF accounted for only $3 \%$, which can be related to low bromide ion levels (mean, $0.24 \mathrm{mg} / \mathrm{L}$ ) in the raw water of TDWTP. The increase in brominated species in the presence of high bromide ion level in water being chlorinated can be attributed to higher reactivity and haloform substitution efficiency of bromide ion (Westerhoff et al., 2004). Distribution of HAN species for tap water from Tahtalı and Balçova reservoirs along with Urla are shown in Fig. 4. As in the case for THM speciation in Urla, brominated compounds, DBAN and BCAN, were found to be the dominant species which constituted $97 \%$ and $2 \%$ of the THAN, respectively. However, due to low bromide ion level $(<0.25 \mathrm{mg} / \mathrm{L})$ in both Tahtalı and Balçova

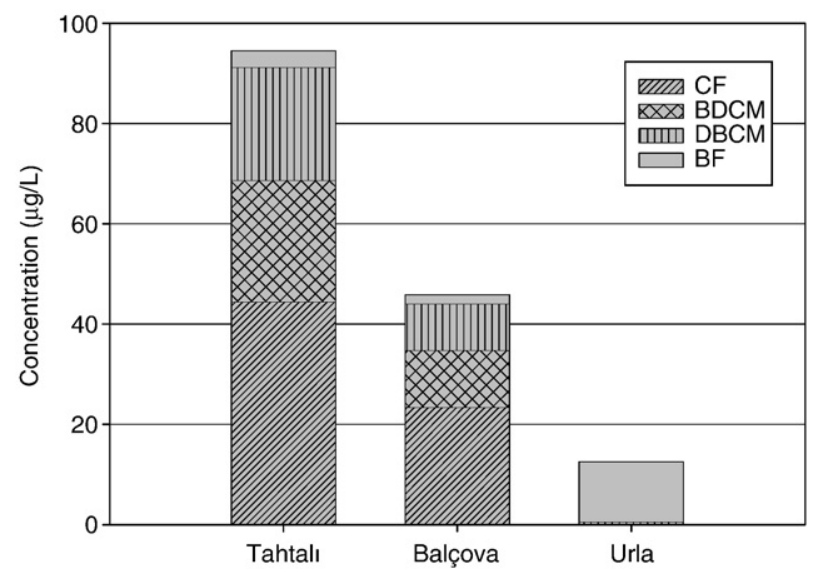

Fig. 3-Species distribution of THMs across water sources. reservoirs, DCAN was found to be the major HAN specie, as suggested by Kim et al. (2002).

\subsection{Seasonal variation in DBP concentrations}

In order to investigate the seasonal variation in DBP concentrations, tap water samples were collected during summer (July and August), fall (September, October, November), winter (December, January, February) and spring (March and April). The seasonal variations in TTHM and THAN concentrations for the three water sources are illustrated in Fig. 5.

The highest TTHM concentrations were measured in spring (mean, $15.4 \mu \mathrm{g} / \mathrm{L}$ ) whereas the lowest were measured in summer and fall (mean; $10.0 \mu \mathrm{g} / \mathrm{L}$ and $11.8 \mu \mathrm{g} / \mathrm{L}$, respectively) at Urla sampling point. Similar trend was observed for TDWTP, as the highest THM occurrence being at Hatay in spring (mean, $134 \mu \mathrm{g} / \mathrm{L}$ ) and the lowest being in summer (mean, $78.8 \mu \mathrm{g} / \mathrm{L})$. The increase in THM formation during spring was mainly due to relatively high raw water NPOC concentration $(4.00 \mathrm{mg} / \mathrm{L})$ compared to the other seasons $(<3.76 \mathrm{mg} / \mathrm{L})$. In addition, TTHM levels in winter were found to be higher than in summer, which is an unexpected situation since many studies reported maximum THM formation in summer (Williams et al., 1998; Rodriguez et al., 2004; Toroz and Uyak, 2005). High THM formation in winter may be related to

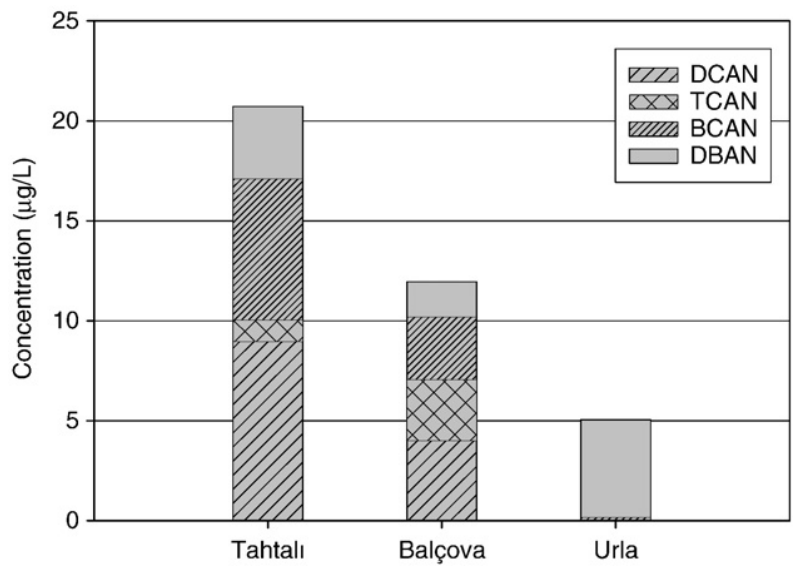

Fig. 4-Species distribution of HANs across water sources. 

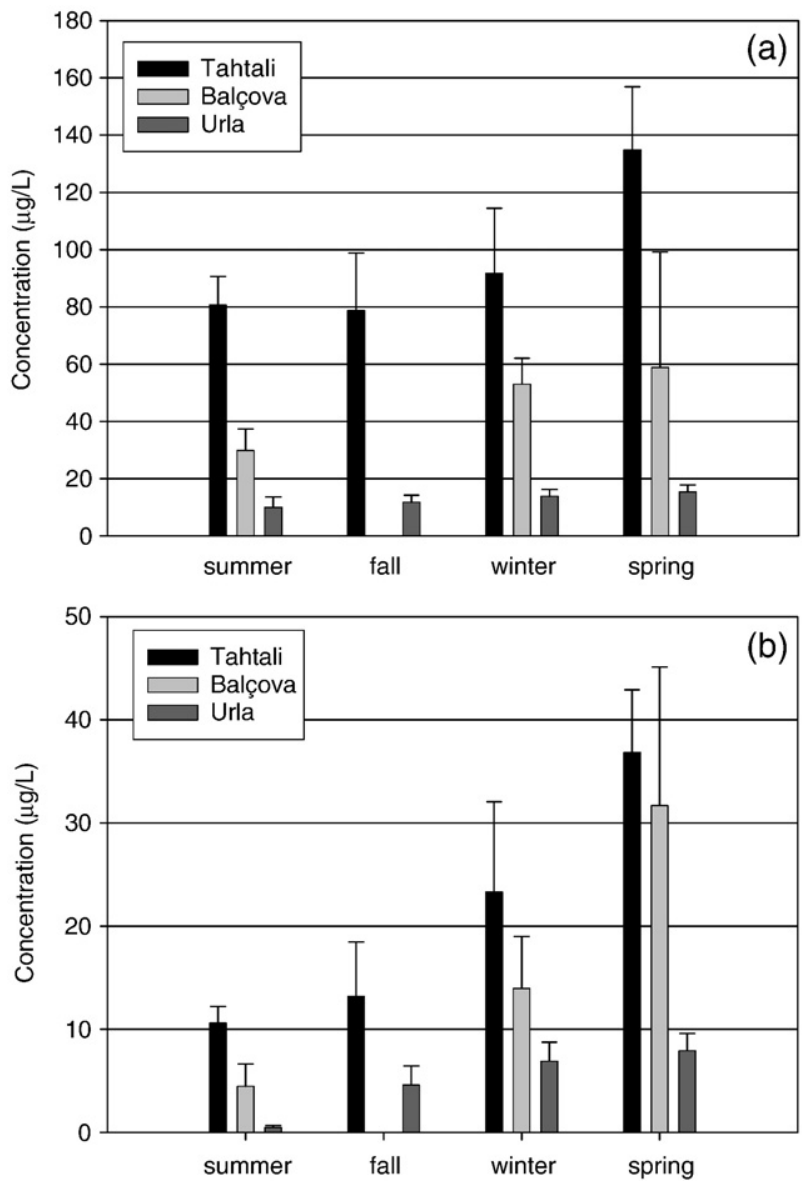

Fig. 5-Seasonal variation in (a) TTHM (b) THAN concentrations in the distribution system of TDWTP, BDWTP and Urla (error bars show one standard deviation).

relatively higher treated water NPOC concentrations (mean, $3.32 \mathrm{mg} / \mathrm{L}$ ) compared to summer (mean, $3.08 \mathrm{mg} / \mathrm{L}$ ) among other possible factors. In support, Ates et al. (2007) reported higher THM concentrations in water samples collected during winter from Balçova and Tahtalı reservoirs than in summer, chlorinated in laboratory conditions at constant temperature.

In the case of BDWTP, which generally supplies drinking water for only two or three seasons, the trend for seasonal TTHM variability was similar to that of Tahtall. BDWTP served for only July, August, December, January and February months in the 10 month study period. TTHM levels were lower for Balçova compared to Tahtall since both pre- and postchlorination doses were much lower in BDWTP, although the NPOC levels of raw and treated waters of the two plants were comparable.

Kruskal-Wallis test was used to examine whether the seasonal variation in THM concentrations was statistically significant. For TDWTP and BDWTP, $p$-values for all THMs were found to be lower than 0.05 suggesting significant variability with seasons. In the case of Urla, the $p$-values for all detected compounds were below 0.003 except for CF $(p=0.33)$, indicating that seasonal variation was not significant for only CF concentration. As seen in Fig. 5, the seasonal trend for THAN concentrations was similar to TTHM, increasing from summer to spring. However, the seasonal differences in
THAN concentrations were found to be higher than TTHM. Kruskal-Wallis test resulted in lower $p$-values for THAN $(<0.001,0.001$, and $<0.001$ for TDWTP, BDWTP, and Urla, respectively) indicating that the differences in THAN concentrations were more significant than TTHM.

\subsection{Regulatory compliance of DBPs}

Evaluation of the temporal variation in TTHM concentrations suggested that DBP concentrations not only showed seasonal variation, but also important intra-seasonal variation was evident depending on variation in the operating conditions. Therefore, at each sampling location, compliance with regulations was assessed using both the highest and lowest sample values within a season as suggested by Rodriguez et al. (2004). The annual average TTHM concentrations calculated using the lowest, the highest, and the mean levels in each season are given in Table 5. The percent difference between the lowest and the highest annual average concentrations was found to be the lowest for Hatay (51\%) and the highest for Balçova (75\%). At Hatay sampling point, while the lowest TTHM level was below the MCL of $80 \mu \mathrm{g} / \mathrm{L}$, the mean and the highest levels were found to be higher than the MCL. All of the annual average TTHM levels for Balçova and Narlıdere sampling points were below the MCL of the USEPA. The highest annual average TTHM level at Güzelbahçe was found to be higher than $80 \mu \mathrm{g} / \mathrm{L}$. When we compared the annual average TTHM concentrations with the MCL stated in Turkish drinking water regulations, all levels complied with the current standard. However, the highest annual average level at Hatay exceeded the MCL of $100 \mu \mathrm{g} / \mathrm{L}$, which will come into effect by the year 2012 .

\subsection{Spatial variation in DBP concentrations}

Balçova, Narlıdere and Güzelbahçe sampling points were on the main distribution line, while $4.5 \mathrm{~km}$ separated the first and second sampling points; there is $6.6 \mathrm{~km}$ between the second and third sampling points. There was a booster chlorination in between the latter sampling points. The highest THM and HAN concentrations were measured in Güzelbahçe, which

Table 5-Evaluation of regulatory compliance of TTHM concentrations

\begin{tabular}{llcrccc}
$\begin{array}{l}\text { Sampling } \\
\text { point }\end{array}$ & & Summer & Fall & Winter & Spring & $\begin{array}{c}\text { Annual } \\
\text { average }\end{array}$ \\
\hline Hatay & H & 94.6 & 128.8 & 125 & 183.0 & 132.8 \\
& L & 59.4 & 57.1 & 40 & 106.1 & 65.6 \\
Balçova & A & 80.6 & 78.7 & 91.8 & 134.9 & 96.5 \\
& H & 49.3 & 87.9 & 77.8 & 55.2 & 67.6 \\
& L & 18.3 & 25.6 & 18.6 & 2.86 & 16.3 \\
Narlidere & A & 28.9 & 60.9 & 49.4 & 18.1 & 39.3 \\
& H & 46.8 & 81.3 & 79.4 & 42.2 & 62.4 \\
& L & 20.0 & 19.4 & 38.0 & 3.15 & 20.1 \\
Güzelbahçe & A & 28.3 & 53.2 & 54.0 & 14.4 & 37.5 \\
& H & 59.3 & 131.3 & 129.6 & 52.8 & 93.2 \\
& L & 16.7 & 28.1 & 41.6 & 10.4 & 24.2 \\
& A & 38.4 & 66.4 & 61.9 & 16.6 & 45.8 \\
\hline
\end{tabular}

Note: the measured samples with the H: highest, L: lowest and A: average concentrations within a season, all concentrations are in $\mu \mathrm{g} / \mathrm{L}$. 


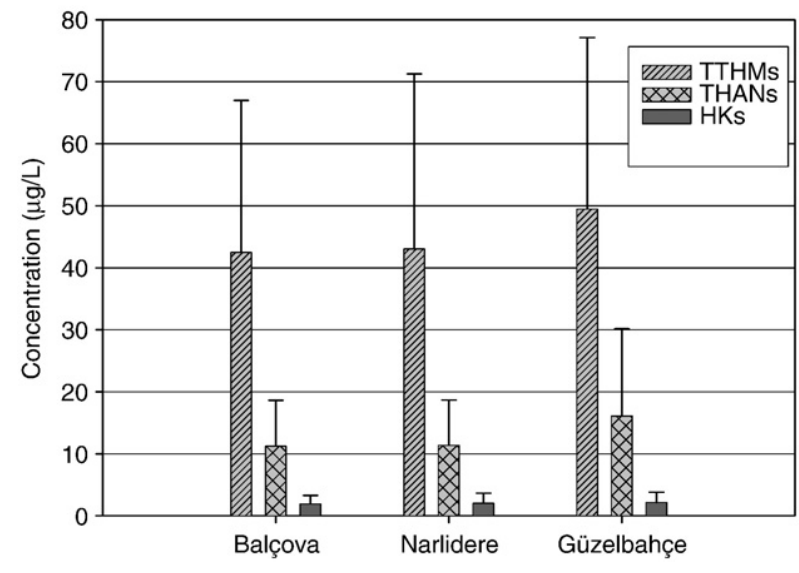

Fig. 6-Spatial variation of DBPs (error bars show one standard deviation).

represented the system extremity, and the lowest in Balçova (Fig. 6). However, Kruskal-Wallis test indicated that the difference in DBP concentrations were not significant except for DBCM and BF. The comparison of the DBP concentrations at the three sampling locations individually showed that the spatial variation in concentrations of TTHM, THAN, and HK were not significant $(p>0.05)$. Although the mean TTHM and THAN concentrations at Balçova and Narlidere were comparable, re-chlorination of drinking water between Narlıdere and Güzelbahçe sampling points resulted in $15 \%$ and $42 \%$ increase in TTHM and THAN levels, respectively. These findings were also in accordance with the other studies that reported increasing levels of DBPs with residence time (Lebel et al., 1997; Rodriguez and Serodes, 2001).

Concentration of free residual chlorine (FRC) was also measured throughout the sampling period at each sampling point. The box-plots of FRC concentrations for the five sampling locations are presented in Fig. 7. At Hatay, concentration of FRC was very low possibly due to high DBP formation, which resulted in high chlorine consumption. The mean FRC concentrations in Balçova, Narlıdere and Güzelbahçe were found to be higher than the sufficient level $(0.2 \mathrm{mg} / \mathrm{L})$ for microbial inactivation (USEPA, 2006). In addition, a notable but subtle increase in the FRC concentration was observed in Güzelbahçe due to re-chlorination of drinking water before this sampling location. However, the subtle increase pointed out that re-chlorination was not very effective. In summary, the results imply that the majority of DBPs were formed by the first sampling point (Balçova), since FRC levels did not vary spatially.

\subsection{Risk assessment}

The carcinogenic and non-carcinogenic risks from exposure to THMs via drinking water ingestion route were estimated in our previous study (Kavcar et al., 2006). THM concentrations were measured at houses of participants who reported daily drinking water intake and body weight by self-administered questionnaires. Population risk levels were estimated by probabilistic approach using Monte Carlo simulation method. In the metropolitan area, the median and mean cancer risks of $\mathrm{BDCM}$ and DBCM were higher than the de minimis level of one in a million $\left(1.0 \times 10^{-6}\right)$. While mean and median cancer risks for $\mathrm{BF}$ were lower than $1.0 \times 10^{-6}$, 95th percentile cancer risk was above $1.0 \times 10^{-6}$. The study also showed that cancer risks from exposure to drinking water contaminants through oral ingestion may be higher than the acceptable level although the concentrations fall below the standards. However, the sampling campaign lasted from August to December representing average conditions but not reflecting seasonal variation.

In this study, we re-estimated the risk levels for tap water in the metropolitan area considering the seasonal concentration variation. Drinking water intake rate and body weight distributions were obtained from the previous study, however, new probability distributions were constructed for the pollutant concentrations based on the enhanced concentration data set that was formed by combining the concentration data from the former and current studies. Descriptive statistics of the corresponding risk levels estimated in the both previous and current studies are presented in Table 6. The median and mean population cancer risk levels were higher than $1.0 \times 10^{-6}$ in the metropolitan area for all three brominated THMs. The median risk levels were at least four times higher than those of the previous study. The differences establish that accounting seasonal variation in DBP concentrations is of utmost importance for human health.

Tokmak et al. (2004) reported that the average multipathway cancer risk for TTHM measured in Ankara tap water was higher than the acceptable risk level. Uyak (2006) reported that ingestion route cancer risks for tap water $\mathrm{CF}, \mathrm{BDCM}$, and DBCM in Istanbul were higher than the acceptable level. Among these compounds, highest cancer risk was calculated for BDCM as $2.1 \times 10^{-5}$ in Esenyurt district. However, these studies did not consider the variation in type of water drunk, drinking water consumption rate, and body weight in the population. Actually, Kavcar et al. (in press) found that $36 \%$ of the metropolitan İzmirians preferred buying spring water to drink in 19-L bottles to tap water. In addition, the slope factor for chloroform was withdrawn by the USEPA concluding that oral RfD is sufficiently protective (IRIS, 2007). Therefore, cancer risk estimates that consider chloroform

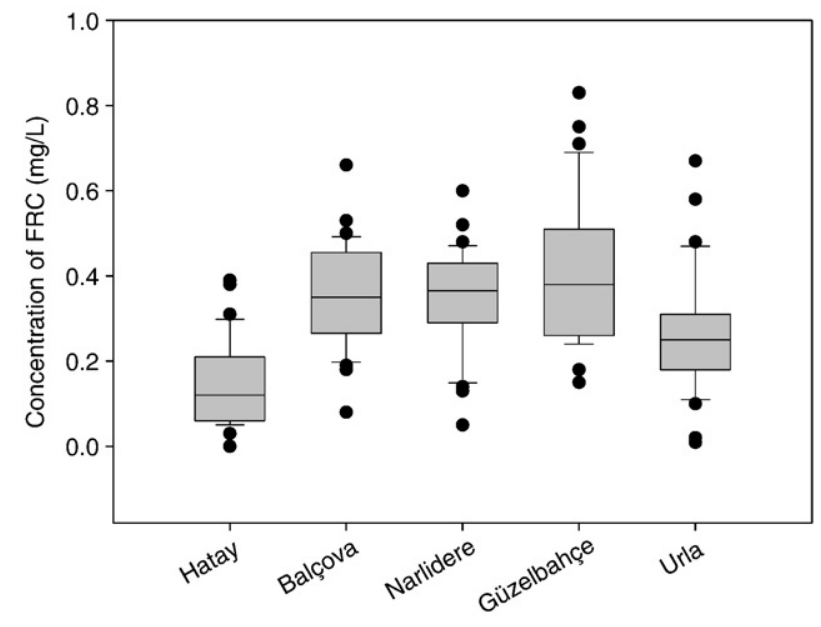

Fig. 7-The box-plots of FRC concentrations for the five sampling locations (the bars above and below the box indicate the 90 th and 10 th percentiles, $\bullet$ points are the measured values outside this range). 
Table 6 - Descriptive statistics for probabilistic carcinogenic risk assessment

\begin{tabular}{|c|c|c|c|c|c|c|c|}
\hline & Mean & Median & $\mathrm{SD}^{\mathrm{b}}$ & Min & $\operatorname{Max}$ & 90th percentile & 95th percentile \\
\hline \multicolumn{8}{|c|}{ Previous data ${ }^{a}$} \\
\hline BDCM & $1.59 \mathrm{E}-05$ & $4.94 \mathrm{E}-06$ & $2.55 \mathrm{E}-05$ & $3.50 \mathrm{E}-23$ & $5.36 \mathrm{E}-04$ & $4.58 \mathrm{E}-05$ & $6.27 \mathrm{E}-05$ \\
\hline DBCM & $1.55 \mathrm{E}-05$ & $4.74 \mathrm{E}-06$ & $3.08 \mathrm{E}-05$ & 7.70E-09 & $5.23 \mathrm{E}-04$ & $4.15 \mathrm{E}-05$ & $6.41 \mathrm{E}-05$ \\
\hline $\mathrm{BF}$ & $3.20 \mathrm{E}-07$ & $1.72 \mathrm{E}-07$ & $4.42 \mathrm{E}-07$ & $2.91 \mathrm{E}-12$ & $1.11 \mathrm{E}-05$ & 7.87E-07 & $1.14 \mathrm{E}-06$ \\
\hline \multicolumn{8}{|c|}{ Enhanced Data } \\
\hline BDCM & $2.50 \mathrm{E}-05$ & $1.63 \mathrm{E}-05$ & $2.83 \mathrm{E}-05$ & $3.93 \mathrm{E}-08$ & $4.72 \mathrm{E}-04$ & $5.52 \mathrm{E}-05$ & $7.65 \mathrm{E}-05$ \\
\hline DBCM & $3.49 \mathrm{E}-05$ & $1.87 \mathrm{E}-05$ & 4.83E-05 & $9.17 \mathrm{E}-10$ & $7.45 \mathrm{E}-04$ & $8.69 \mathrm{E}-05$ & $1.19 \mathrm{E}-04$ \\
\hline $\mathrm{BF}$ & $6.63 \mathrm{E}-06$ & $4.64 \mathrm{E}-06$ & $6.86 \mathrm{E}-06$ & $5.66 \mathrm{E}-10$ & 1.27E-04 & 1.42E-05 & $1.87 \mathrm{E}-05$ \\
\hline $\begin{array}{l}\text { All risk } \\
\text { a Kavc } \\
\text { b Stan }\end{array}$ & $\begin{array}{l}\text { are for } \mathrm{m} \\
\text { (2006). } \\
\text { viation. }\end{array}$ & rea & & & & & \\
\hline
\end{tabular}

are over estimations. Furthermore, health risk levels calculated for individual compounds should not, simply, be summed to report a total risk level (Butterworth, 2005).

The carcinogenic and non-carcinogenic risk levels were also separately estimated for surface and groundwaters, and presented in Fig. 8. All carcinogenic risks were higher than $1.0 \times 10^{-6}$, except for $\mathrm{BF}\left(8.2 \times 10^{-7}\right)$ for surface water. Both carcinogenic and non-carcinogenic risks for $\mathrm{BF}$ were found to be higher for groundwater because the measured concentrations were much higher in Urla. Hsu et al. (2001), also, estimated higher risk levels for $\mathrm{BF}$ in well-water compared to surface water. They reported
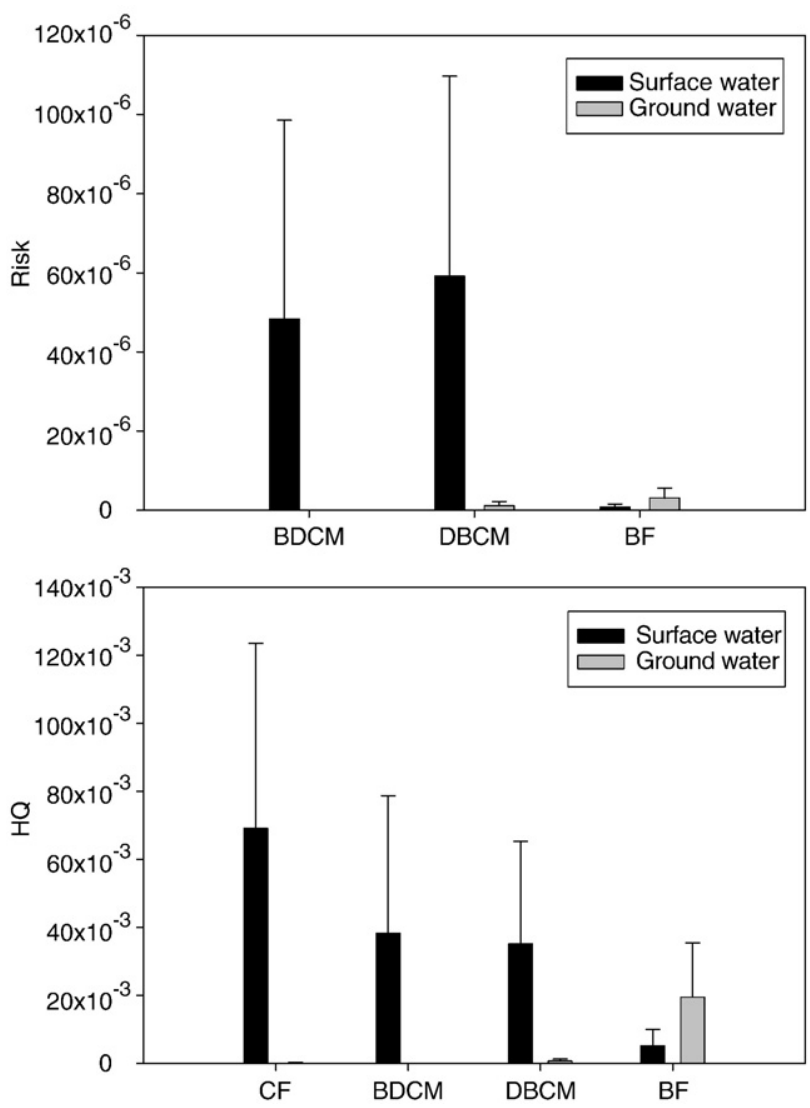

Fig. 8-Comparison of a) carcinogenic b) non-carcinogenic risks for surface and ground water categories (error bars show one standard deviation). that cancer risk levels for BF (based on $2 \mathrm{~L}$ water/day) as $0.28 \times 10^{-6}$ for surface and $0.39 \times 10^{-6}$ for well water in Southern Taiwan.

\section{Summary and conclusions}

Four THMs (CF, BDCM, DBCM, and BF) were the most abundant DBPs, while HKs (1,2-DCP and 1,1,1-TCP) were detected at the lowest concentrations. The level of DBP concentrations as well as the species distribution was differed by the type of source water. Higher DBP concentrations were measured in surface water supplied tap waters, and chlorinated DBPs were the predominant species, whereas in groundwater supplied tap waters, brominated ones dominated the distribution. The seasonal variation in concentrations was significant for all DBPs except for THAN in the distribution system of Balçova, and CF in Urla. The highest DBP levels were detected in spring while the lowest were measured during summer and fall. Annual average TTHM levels at all sampling locations were in attainment of the drinking water standards of Turkey and the USEPA, except for Hatay district. The concentrations of DBPs increased in the distribution system as the residence time increased. The highest DBP formation was observed in Güzelbahçe district, which represented the system extremity.

While the median and mean cancer risks for all THM species were higher than $1.0 \times 10^{-6}$, non-carcinogenic risks due to exposure to THMs in İzmir drinking water were negligible. DBCM and BDCM carcinogenic risk levels were much higher than that of BF. When the effect of source water is considered, both cancer and non-cancer risks for BF was higher for groundwater while those for DBCM, BDCM, and CF were higher for surface water. The differences in cancer risk levels estimated with the previous and enhanced concentration data sets established that seasonal and source effects must be considered in human health risk assessment studies.

\section{Acknowledgements}

The authors would like to thank Izmir Water and Sewerage Works Authority (IZSU) for providing the NPOC and bromide samples, and temperature and $\mathrm{pH}$ data. Environmental Research Center of İmir Institute of Technology is gratefully acknowledged for sample analyses. 


\section{R E F E R E N C E S}

Ates N, Kaplan SS, Sahinkaya E, Kitis M, Dilek FB, Yetis U. Occurrence of disinfection by-products in low DOC surface water in Turkey. J Hazard Mater 2007;142:526-34.

Butterworth BE. Science based risk assessments for drinking water disinfection by-products. Environ Res 2005;98:276-8.

Calderon RL. The epidemiology of chemical contaminants of drinking water. Food Chem Toxicol 2000;38:S13-20.

Glezer V, Harris B, Tal N, Iosefzon B, Lev O. Hydrolysis of haloacetonitriles: linear free energy relationship, kinetics and products. Water Res 1998;33:1938-48.

Golfinopoulos SK, Nikolaou AD. Survey of disinfection by-products in drinking water in Athens, Greece. Desalination 2005;176:13-24.

Heller L, Grossman L. Formation of cynogen bromide and other volatile DBPs in the disinfection of bromide rich lake water. Environ Sci Technol 1999;33:932-7.

Hsu CH, Jeng WL, Chang RM, Chien LC, Han BC. Estimation of potential lifetime cancer risks for trihalomethanes from consuming chlorinated drinking water in Taiwan. Environ Res 2001;85:77-82.

IARC. Monograms on the Evaluation of carcinogenic Risk to Human: Chlorinated Drinking water; Chlorination By-products; Some Other Halogenated Compounds; Cobalt and Cobalt Compounds. WHO; 1991. p. 52.

IRIS. Integrated Risk Information System of U.S. Environmental Protection Agency; 2007. http://www.epa.gov/iris.

Kampioti AA, Stephanou EG. The impact of bromide on the formation of neutral and acidic disinfection by-products in Mediterranean chlorinated drinking water. Water Res 2002;36:2596-606.

Kavcar P, Odabasi M, Kitis M, Inal F, Sofuoglu SC. Occurrence, oral exposure and risk assessment of VOCs in drinking water for İzmir. Water Res 2006;40:3219-30.

Kavcar P, Sofuoglu A, Sofuoglu SC. A health risk assessment for exposure to trace metals via drinking water ingestion pathway. Int J Hyg Environ Health 2008. doi:10.1016/j.jheh.2008.05.002.

Kawamoto T, Makihata N. Distribution of bromine/ chlorine-containing disinfection by-products in tap water from different water sources in the Hyogo prefecture. J Health Sci 2004;50:235-47.

Kim J, Chung Y, Shin Dongchun S, Kim M, Lee Y, Lim Y, et al. Chlorination by-products in surface water treatment process. Desalination 2002;151:1-9.

Lebel GL, Benoit FM, Williams DT. A one year survey of halogenated disinfection by-products in the distribution system of treatment plants using three different disinfection processes. Chemosphere 1997;34:2301-8.

Lee SC, Guo H, Lam SMJ, Lau LA. Multipathway risk assessment on disinfection by-products of drinking water in Hong Kong. Environ Res 2004;94:47-56.

Liang L, Singer PC. Factors influencing the formation and relative distribution of haloacetic acids and trihalomethanes in drinking water. Environ Sci Technol 2003;37:2920-8.

Muellner MG, Wagner ED, Mccalla K, Richardson S, Woo Y, Plewa MJ. Haloacetonitriles vs. regulated haloacetic acids: are nitrogen containing DBPs more toxic? Environ Sci Technol 2007;41:645-51.

Rodriguez MJ, Serodes JB. Spatial and temporal evolution of trihalomethanes in three water distribution systems. Water Res 2001;35:1572-86.

Rodriguez MJ, Vinnete Y, Serodes JB, Bouchard C. Trihalomethanes in drinking water of Greater Quebec Region (Canada): occurrence, variations and modeling. Environ Monit Assess 2003;89:69-93.
Rodriguez MJ, Serodes JB, Levallois P. Behavior of trihalomethanes and haloacetic acids in a drinking water distribution system. Water Res 2004;38:4367-82.

Simpson KL, Hayes PH. Drinking water disinfection by-products: an Australian perspective. Water Res 1998;32:1522-8.

Sofuoglu SC, Lebowitz MD, O’Rourke MK, Robertson GL, Dellarco M, Moschandreas DJ. Exposure and risk estimates for Arizona drinking water. J Am Water Works Assoc 2003;95:67-79.

Tokmak B, Capar G, Dilek FB, Yetis U. Trihalomethanes and associated potential cancer risks in the water supply in Ankara, Turkey. Environ Res 2004;96:345-52.

Toroz I, Uyak V. Seasonal variations of trihalomethanes (THMs) in water distribution networks of Istanbul City. Desalination 2005;176:127-41.

Turkish Ministry of Health. Insani tüketim amaçlı sular hakkında yönetmelik (in Turkish). Off J 2005:25730 Ankara.

USEPA. Guidelines for exposure assessment. Risk Assessment Forum, US Environmental Protection Agency; 1992. Washington DC, EPA/600/Z-92/001.

USEPA. Method 551.1. Determination of chlorination disinfection by-products, chlorinated solvents, and halogenated pesticides/ herbicides in drinking water by liquid-liquid extraction and gas chromatography with with electron capture detection. Environmental Monitoring System Laboratory. Cincinnati, Ohio: Office of Research and Development, US Environmental Protection Agency; 1995.

USEPA. Exposure Factors Handbook. Office of Research and Development, National Center for Environmental Assessment, US Environmental Protection Agency; 1997. Washington, DC, EPA/600/P-95/002Fa.

USEPA. National Primary Drinking Water Regulations: Disinfectants and Disinfection Byproducts Final Rule. 1998; 40 CFR Parts 9, 14, and 142. Federal Register Part IV, 63: 241, 69389- 69476.

USEPA. Guidance for Performing Aggregate Exposure and Risk Assessments. Office of Pesticide Programs, US Environmental Protection Agency; 1999. Washington, DC.

USEPA. Guidelines For Carcinogen Risk Assessment. Risk Assessment Forum, US Environmental Protection Agency; 2005. Washington, DC. EPA/630/P-03/001F.

USEPA. National Primary Drinking Water Regulations: Disinfectants and Disinfection Byproducts Final Rule 2006; 40 CFR Parts 9, 141, and 142. Federal Register Part II, 71: 387-493.

Uyak V. Multipathway risk assessment of trihalomethanes exposure in Istanbul drinking water supply. Environ Int 2006;32:12-21.

Villanova RJG, Garcia C, Gomez JA, Garcia MP, Ardanuy R. Formation, evolution and modeling of trihalomethanes in the drinking water of a town: II. In the distribution system. Water Res 1997;31:1405-23.

Villanueva CM, Kogevinas M, Grimalt JO. Haloacetic acids and trihalomethanes in finished drinking water from heterogeneous sources. Water Res 2003;37:953-8.

Villanueva CM, Cantor PK, Cordier S, Jakkola JJK, King WD, Lynch FC, et al. Disinfection by-products and bladder cancer. Epidemiology 2004;5:357-67.

Wang GS, Chen DY, Lin TF. Cancer risk assessment from trihalomethanes in drinking water. Sci Total Environ 2007;387 (15):86-95.

Westerhoff P, Chao P, Mash H. Reactivity of natural organic matter with aqueous chlorine and bromine. Water Res 2004;38:1502-13.

Williams DT, Benoit FM, Lebel GL. Trend in levels of disinfection by-products. Environmetrics 1998;9:555-63. 